\title{
Multi-layered GMDH-type Neural Network Algorithm Using Principal Component-Regression Analysis and PSS Criterion
}

\author{
Tadashi Kondo ${ }^{1)}$, Junji Ueno and Shoichiro Takao \\ Graduate School of Health Sciences, The University of Tokushima \\ 3-18-15 Kuramoto-cho Tokushima, 770-8509 Japan \\ ${ }^{1)}$ E-mail: kondo@medsci.tokushima-u.ac.jp
}

\begin{abstract}
In this study, a hybrid Group Method of Data Handling (GMDH)-type neural network algorithm using principal component-regression analysis is proposed and applied to the nonlinear system identification. The architectures of the GMDH-type neural networks are automatically organized using heuristic self-organization method which is a kind of evolutionary computation.In the heuristic self-organization method, many nonlinear combinations of the input variables are generated and new neurons are constructed from these combinations. Only desirable neurons which fit the characteristics of the nonlinear system, are selected and these neurons are combined again in next layer. These procedures are iterated and a multi-layered neural network architectures are automatically organized. In the GMDH-type neural network, the multi-colinearity of the variables generates and the prediction output values of the neural network become unstable. In this study, the principal component-regression analysis is used for estimating the parameters of the neurons and stable and accurate multi-layered architectures of the GMDH-type neural networks are organized using the heuristic self-organization.
\end{abstract}

\section{Introduction}

In this study, a revised Group Method of Data Handling (GMDH)-type neural network algorithm using principal component-regression analysis is proposed and applied to the nonlinear system identification. The GMDH-type neural network algorithms and their applications have been proposed in our early works [1]-[4]. GMDH-type neural networks can automatically organize neural network architecture by heuristic self-organization method [5],[6] and they can also determine such structural parameters as the number of layers, the number of neurons in hidden layers and useful input variables so as to minimize the prediction error criterion defined as Akaike's information criterion (AIC) [7] or Prediction sum of squared (PSS) [8]. The heuristic self-organization method can be used for organizing the multi-layered architecture but the multicolinearity in the partial polynomials of the neurons is generated. The nonlinear models which contain the multicolinearity in the partial polynomials is very unstable and can not be used in the prediction problem. In the conventional GMDH-type neural networks [1]-[4], the stepwise regression analysis [9] is used to avoid the multicolinearity and stable parameters in the neurons can be estimated. In this paper, the revised GMDH-type neural network algorithm is proposed and the principal component-regression analysis [10] is used for estimating the parameters of the neurons and stable and accurate multi-layered architectures of the GMDH-type neural networks are organized using the heuristic self-organization. This algorithm is applied to the identification problem of the nonlinear system and the identification results are compared with feedback GMDH-type neural network [3], GMDH [8] and conventional neural network trained using the back propagation algorithm. It is shown that the revised GMDH-type neural network algorithm is very accurate for the nonlinear system identification.

\section{Heuristic self-organization}

Architectures of GMDH-type neural network are automatically organized by the heuristic self-organization method. First, the procedures of the heuristic self-organization method are shown because it plays very important roles for organization of GMDH-type neural network. Heuristic self-organization method is a kind of the evolutionary computation.

Heuristic self-organization method is constructed by the following six procedures:

1) Separating original data into training and test sets

Original data is separated into training and test sets. Training data is used for estimating parameters of partial descriptions which describe partial relationships of the nonlinear system. Test data is used for organizing complete description which describes complete relationships between input and output variables of the nonlinear system.

2) Generating combinations of input variables in each layer

All combinations of two input variables $\left(x_{i}, x_{j}\right)$ are generated in each layer. The number of combinations is $\frac{p !}{(p-2) ! 2 !}$. Here, $p$ is the number of input variables.

3) Calculating partial descriptions 
For each combination, partial descriptions of the nonlinear system can be calculated by applying regression analysis to training data. Output variables of partial descriptions are called as intermediate variables.

\section{4) Selecting intermediate variables}

$L$ intermediate variables which give $L$ smallest test errors calculated using test data are selected from generated intermediate variables.

\section{5) Iterating calculations from procedure 2) to 4)}

Selected $L$ intermediate variables are set to input variables of the next layer and calculations from procedure 2) to 4) are iterated. The multi-layered architecture is organized.

\section{6) Stopping multi-layered iterative calculations}

When errors of test data in each layer stop decreasing, iterative calculation is terminated. Finally, complete description of the nonlinear system is organized by partial descriptions generated in each layer.

\section{Revised GMDH-type neural network algorithm}

The revised GMDH-type neural network has a common feedforward multilayered architecture. Figure 1 shows architecture of the revised GMDH-type neural network. This neural network is organized by the heuristic self-organization method.

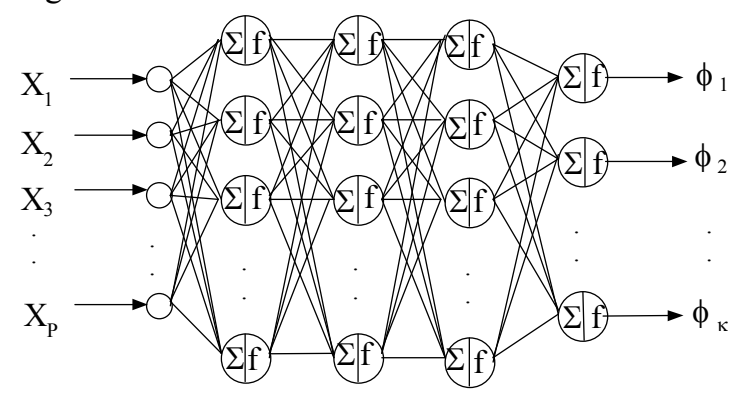

Fig.1 Architecture of revised GMDH-type neural network

Procedures for determining architecture of revised GMDH-type neural network conform to the following:

\subsection{The first layer}

$$
u_{j}=x_{j} \quad(j=1,2, \ldots, p)
$$

where $x_{j}(j=1,2, \ldots, p)$ are input variables of the nonlinear system, and $p$ is the number of input variables. In the first layer, input variables are set to output variables.

\subsection{The second layer}

All combinations of $r$ input variables are generated. For each combination, optimum neuron architectures are automatically selected from the following two neurons.

Architectures of the first and second type neurons are shown in Fig. 2 and Fig.3 respectively. Optimum neuron architecture for each combination is selected from the first and second type neuron architectures.

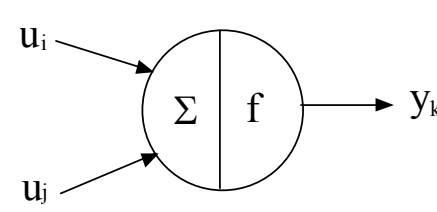

Fig.2 Neuron architecture of the first type neuron

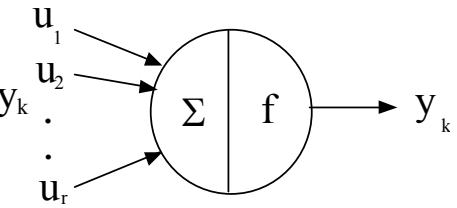

Fig.3 Neuron architecture of the second type neuron
Revised GMDH-type neural network algorithm can select optimum neural network architecture from three neural network architectures such as sigmoid function neural network, RBF neural network and polynomial neural network.

\subsubsection{Neuron architectures}

Neuron architectures of the first and second type neurons in each neural network architecture are shown as follows

1) Sigmoid function neuron

a) The first type neuron

$\Sigma$ : (Nonlinear function)

$$
z_{k}=w_{1} u_{i}+w_{2} u_{j}+w_{3} u_{i} u_{j}+w_{4} u_{i}^{2}+w_{5} u_{j}^{2}-w_{0} \theta_{l}
$$

$\mathrm{f}$ : (Nonlinear function)

$$
y_{k}=\frac{1}{1+e^{\left(-z_{k}\right)}}
$$

Here, $\theta_{l}=1$ and $w_{i}(i=0,1,2, \ldots, 5)$ are weights between the first and second layer. Value of $r$, which is the number of input variables $u$ in each neuron, is set to two for the first type neuron.

\section{b) The second type neuron}

$\Sigma$ : (Linear function)

$$
z_{k}=w_{1} u_{1}+w_{2} u_{2}+w_{3} u_{3}+\cdots+w_{r} u_{r}-w_{0} \theta_{l} \quad(r<p)
$$

$\mathrm{f}$ : (Nonlinear function)

$$
y_{k}=\frac{1}{1+e^{\left(-z_{k}\right)}}
$$

Here, $\theta_{l}=1$ and $w_{i}(i=0,1,2, \ldots, r)$ are weights between the first and second layer. Value of $r$, which is the number of input variables $u$ in each neuron, is set to be greater than two and smaller than $p$ for the second type neuron. Here $p$ is the number of input variables $x_{i} \quad(i=1,2, \ldots, p)$.

2) Radial basis function neuron

a) The first type neuron

$\Sigma$ : (Nonlinear function)

$$
z_{k}=w_{1} u_{i}+w_{2} u_{j}+w_{3} u_{i} u_{j}+w_{4} u_{i}^{2}+w_{5} u_{j}^{2}-w_{0} \theta_{l}
$$

$\mathrm{f}:$ (Nonlinear function)

$$
y_{k}=e^{\left(-z_{k}^{2}\right)}
$$

Here, $\theta_{l}=1$ and $w_{i}(i=0,1,2, \ldots, 5)$ are weights between the first and second layer. Value of $r$, which is the number of input variables $u$ in each neuron, is set to two for the first type neuron.

b) The second type neuron

$\Sigma$ : (Linear function)

$$
z_{k}=w_{1} u_{1}+w_{2} u_{2}+w_{3} u_{3}+\cdots+w_{r} u_{r}-w_{0} \theta_{l} \quad(r<p)
$$

$\mathrm{f}:$ (Nonlinear function)

$$
y_{k}=e^{\left(-z_{k}^{2}\right)}
$$

Here, $\theta_{l}=1$ and $w_{i}(i=0,1,2, \ldots, r)$ are weights between the first and second layer. Value of $r$, which is the number of 
input variables $u$ in each neuron, is set to be greater than two and smaller than $p$ for the second type neuron. Here $p$ is the number of input variables $x_{i} \quad(i=1,2, \ldots, p)$.

\section{3) Polynomial neuron}

\section{a) The first type neuron}

$\Sigma$ : (Nonlinear function)

$$
\begin{gathered}
z_{k}=w_{1} u_{i}+w_{2} u_{j}+w_{3} u_{i} u_{j}+w_{4} u_{i}^{2}+w_{5} u_{j}^{2}-w_{0} \theta_{1} \\
\mathrm{f}: \text { (Linear function) } \\
y_{k}=z_{k}
\end{gathered}
$$

Here, $\theta_{l}=1$ and $w_{i}(i=0,1,2, \ldots, 5)$ are weights between the first and second layer. Value of $r$, which is the number of input variables $u$ in each neuron, is set to two for the first type neuron.

\section{b) The second type neuron}

$\Sigma$ : (Linear function)

$$
\begin{aligned}
& z_{k}=w_{1} u_{1}+w_{2} u_{2}+w_{3} u_{3}+\cdots+w_{r} u_{r}-w_{0} \theta_{l} \quad(r<p) \\
& \mathrm{f}: \text { (Linear function) } \\
& y_{k}=z_{k}
\end{aligned}
$$

Here, $\theta_{l}=1$ and $w_{i}(i=0,1,2, \ldots, r)$ are weights between the first and second layer. Value of $r$, which is the number of input variables $u$ in each neuron, is set to be greater than two and smaller than $p$ for the second type neuron. Here $p$ is the number of input variables $x_{i} \quad(i=1,2, \ldots, p)$. Weights $w_{i}(i=0,1,2$,$) in each neural network architecture are$ estimated by the principal component-regression analysis [10].

\subsubsection{Estimation procedure of weight $w_{i}$}

First, values of $z_{k}^{* *}$ are calculated for each neural network architecture as follows.

\section{1) Sigmoid function neural network}

$$
z_{k}^{* *}=\log _{e}\left(\frac{\phi^{\prime}}{1-\phi^{\prime}}\right)
$$

2) $R B F$ neural network

$$
z_{k}^{* *}=\sqrt{-\log _{e} \phi^{\prime}}
$$

\section{3) Polynomial neural network}

$$
z_{k}^{* *}=\phi
$$

where $\phi^{\prime}$ is the normalized output variable whose values are between 0 and 1. $\phi^{\prime}$ is calculated using following equation.

$$
\phi^{\prime}=\frac{\phi-\phi_{\min }}{\phi_{\max }-\phi_{\min }}
$$

Here, $\phi_{\max }$ is the maximum value of the output variable $(\phi)$, and $\phi_{\min }$ is the minimum value of $\phi$.

Weights $w_{i}(i=0,1,2, \ldots)$ in each neural network architecture are estimated by the principal component-regression analysis.

\subsubsection{Principal component-regression analysis.}

Multicolinearity is generated in the function $\Sigma$ of the neurons because heuristic self-organization method is used. In this study, the function $\Sigma$ is calculated using the principal component-regression analysis.

In the case of Eq.(2), orthogonal vector $\underline{v}$ is calculated .

$$
\underline{\boldsymbol{v}}=C \cdot \underline{\boldsymbol{u}}
$$

Here,

$$
\begin{aligned}
& \underline{\boldsymbol{v}}=\left(v_{1}, v_{2}, \ldots, v_{5}\right) \\
& \underline{\boldsymbol{u}}=\left(u_{i} u_{j}, u_{i} u_{j}, u_{i}{ }^{2}, u_{j}{ }^{2}\right)
\end{aligned}
$$

$\underline{\boldsymbol{y}}$ is orthonormal vectors and $C$ is orthonormal matrix. $C$ is calculated using the following eigenvalue problem .

$$
R \cdot C=C \cdot \Lambda
$$

Here, $R$ is a correlation matrix. Then, variable $z_{k}$ is calculated using orthogonal regression analysis.

$$
\begin{aligned}
z_{k} & =\underline{\boldsymbol{w}}^{\mathbf{T}} \cdot \underline{\boldsymbol{v}} \\
& =w_{1} v_{1}+w_{2} v_{2}+\ldots+w_{5} v_{5}
\end{aligned}
$$

Using the principal component-regression analysis, variable $z_{k}$ in the function $\Sigma$ is calculated without multi-colinearity. In (20), useful orthogonal variables $v_{i}(i=1,2, \ldots, 5)$ are selected using PSS criterion[8] as the variable selection criterion [9].

PSS is described by the following equations:

$$
P S S=\sum_{\alpha=1}^{n}\left(z_{\alpha}^{* *}-z_{k \alpha}^{*}\right)^{2}
$$

where

$$
z_{k \alpha}{ }^{*}=w_{1 \alpha} v_{1 \alpha}+w_{2 \alpha} v_{2 \alpha}+\ldots+w_{5 \alpha} v_{5 \alpha} \quad \alpha=1,2, \ldots, n
$$
$n$ is the number of training data, $z_{\alpha}{ }^{* *}$ is the $\alpha$-th observed value of $z^{* *}, v_{i \alpha}$ is the $\alpha$-th observed value for the input variable $\left(v_{i}\right)$, and $z_{k \alpha}{ }^{*}$ is the $\alpha$-th estimated value obtained by the multiple regression analysis of all the data except the $\alpha$-th datum. In order to calculate PSS in (21), the multiple regression analysis must be repeated $n$ times, and the amount of calculations increases in the number of data. But PSS in (21) can be reduced as follows,

$$
P S S=\sum_{\alpha=1}^{n}\left\{\frac{z_{\alpha}^{* *}-z_{k \alpha}}{1-\underline{v}_{\alpha}^{T}\left(V^{T} V\right)^{-1} \underline{v}_{\alpha}}\right\}^{2}
$$

where

$$
\begin{aligned}
& z_{k \alpha}=w_{1} v_{1 \alpha}+w_{2} v_{2 \alpha}+\ldots+w_{5} v_{5 \alpha} \quad \alpha=1,2, \ldots, n \\
& \underline{v}_{\alpha}^{\mathrm{T}}=\left[v_{1 \alpha}, v_{2 \alpha}, \ldots, v_{5 \alpha}\right], \quad \alpha=1,2, \ldots, n \\
& V^{\mathrm{T}}=\left[v_{1}, v_{2}, \ldots, v_{n}\right]
\end{aligned}
$$

$z_{k \alpha}$ is the $\alpha$-th estimated value obtained by the multiple regression analysis of all the data. With this procedure, we need not repeat the regression analysis $n$ times. PSS criterion does not contain the statistical assumption in the regression model.

For each combination, three neuron architectures which are sigmoid function neuron, RBF neuron and polynomial neuron, are generated and $L$ neurons which minimize PSS are selected for each neuron architecture. From these $L$ selected neurons for each neuron architecture, estimation errors of $L$ neurons are calculated. Then, neural network architecture which has minimum estimation error is selected as revised GMDH-type neural network architecture from three neural network architectures such as sigmoid function neural network, RBF neural network and polynomial neural network.

After the type of revised GMDH-type neural network architecture is selected, output variables $y_{k}$ of $L$ selected neurons are set to input variables of neurons in the third layer.

\subsection{The third and successive layers}

In the second layer, optimum neural network architecture is selected from three neural network architectures. In the third and successive layers, only one neuron architecture, which is sigmoid function neuron or 
RBF neuron or polynomial neuron, is used for calculation and the same calculation of the second layer is iterated until PSS values of $L$ neurons with selected neuron architecture stop decreasing. When iterative calculation is terminated, neural network architecture is produced by $L$ selected neurons in each layer.

By using these procedures, the revised GMDH-type neural network using the principal component-regression analysis is organized. Figure 4 shows the flowchart of the revised GMDH-type neural network. Revised GMDH-type neural network has an ability of self-selecting optimum neural network architecture. Therefore, neural network architecture is automatically selected from three neural network architectures. Furthermore, structural parameters such as the number of layers, the number of neurons in hidden layers and useful input variables are automatically selected to minimize prediction error criterion defined as PSS.

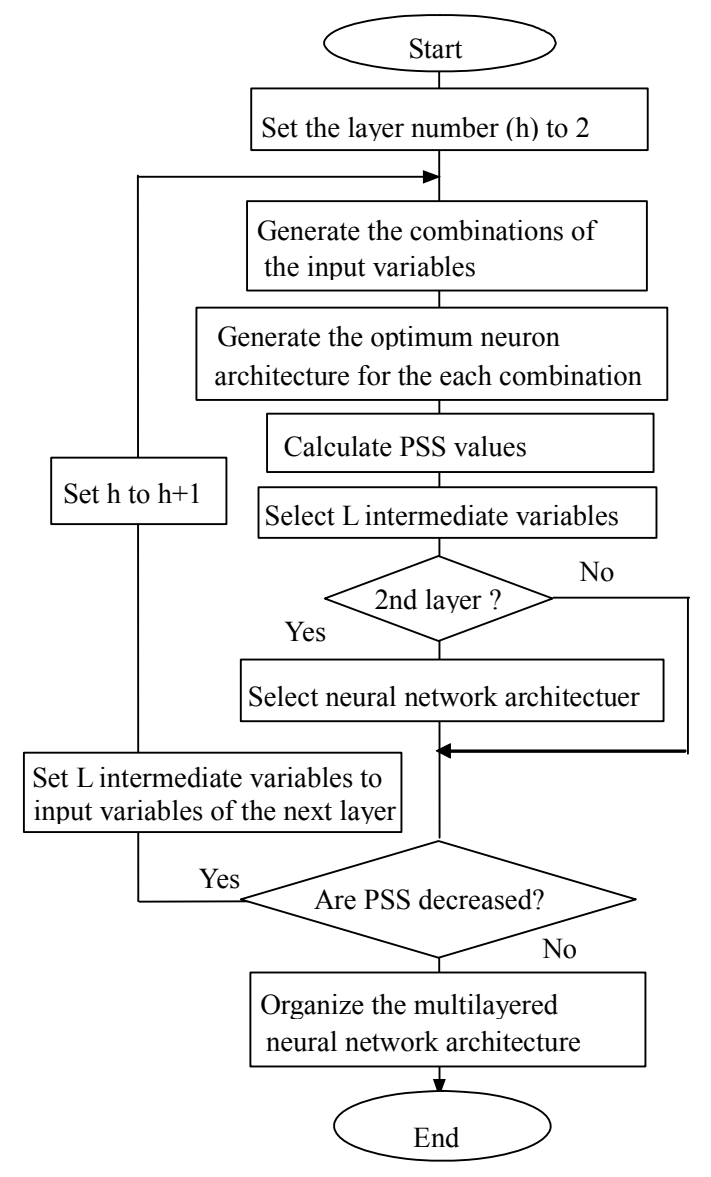

Fig.4 Flowchart of the revised GMDH-type neural network

\section{Application to nonlinear system identification}

To verify the performance of the revised GMDH-type neural network, it is applied to the nonlinear system identification problem. In this application, PSS was used as the prediction error criterion.

\subsection{Nonlinear system identification problem}

The nonlinear system is assumed to be described by the following equations:

$$
\begin{aligned}
\phi_{1} & =\left(1.0+1.1 x_{1}+1.2 x_{2}+1.3 x_{3}\right)^{4}+\varepsilon_{1} \\
\phi_{2} & =\left(1.0+1.4 x_{1}+1.5 x_{2}+1.6 x_{3}\right)^{4}+\varepsilon_{2} \\
\phi_{3} & =\left(1.0+1.7 x_{1}+1.8 x_{2}+1.9 x_{3}\right)^{4}+\varepsilon_{3} \\
\phi_{4} & =\left(1.0+2.0 x_{1}+2.1 x_{2}+2.2 x_{3}\right)^{4}+\varepsilon_{4}
\end{aligned}
$$

Here, $\phi_{1} \sim \phi_{4}$ show output variables and $x_{1} \sim x_{3}$ show input variables. $\varepsilon_{1} \sim \varepsilon_{4}$ are Gaussian white noise, i.e., $\mathrm{N}\left(0,0.005^{2}\right)$. Furthermore, $x_{4}$ is added as the input variable of the neural network in order to check that the revised GMDH-type neural network can eliminate the useless input variables. The neural network is organized by using twenty training data. The prediction is carried out by using twenty testing data so as to check the generalization ability.

\subsection{Identification results obtained using the revised GMDH-type neural network}

\section{1) Input variables}

Four input variables were used but the useless input variables $x_{4}$ was automatically eliminated.

\section{2) Number of selected neurons}

Four neurons were selected in the hidden layer.

3) Selection of the neural network architecture

The polynomial neural network architecture was selected as the revised GMDH-type neural network architecture.

\section{4) Structure of the neural network}

The calculation of the GMDH-type neural network was terminated at the sixth layer.

\section{5) Estimation accuracy}

The estimation accuracy was evaluated by using the following equation:

$$
J_{1}=\frac{\sum_{i=1}^{20}\left|\phi_{i}-\phi_{i}^{*}\right|}{\sum_{i=1}^{20}\left|\phi_{i}\right|}
$$

where $\phi_{i}(i=1,2, \cdots, 20)$ were the actual values and $\phi_{i}{ }^{*}$ $(i=1,2, \cdots, 20)$ were the estimated values by the revised GMDH-type neural network. The values of $J_{1}$ for four output variables are shown in Table1.

\section{6) Prediction accuracy}

The prediction accuracy was evaluated by using the following equation:

$$
J_{2}=\frac{\sum_{i=21}^{40}\left|\phi_{i}-\phi_{i}^{*}\right|}{\sum_{i=21}^{40}\left|\phi_{i}\right|}
$$

where $\phi_{i}(i=21,22, \cdots, 40)$ were the actual values and $\phi_{i}{ }^{*}$ $(i=21,22, \cdots, 40)$ were the predicted values by the revised GMDH-type neural network. The values of $J_{2}$ for four output variables are shown in Table1.

7) Variation of PSS and estimated values 
The variation of PSS in (23) of the output variables $\phi_{1}$ is shown in Fig.5. It decreased gradually through layers and converged at the sixth layer. The estimated values of $\phi_{1}$ by the revised GMDH-type neural network is shown in Fig.6. We can see that the estimated values are very accurate.

Table 1 Prediction and estimation accuracy

\begin{tabular}{|l|l|r|r|r|r|}
\hline Model & $\mathrm{J}$ & $\phi 1$ & $\phi 2$ & $\phi 3$ & $\phi 4$ \\
\hline \multirow{2}{*}{$\begin{array}{l}\text { Revised GMDH-NN } \\
\text { (PSS) }\end{array}$} & $\mathrm{J} 1$ & 0.007 & 0.007 & 0.007 & 0.007 \\
\hline Feedback GMDH-NN & $\mathrm{J} 2$ & 0.011 & 0.009 & 0.009 & 0.008 \\
\cline { 2 - 7 } (PSS) & $\mathrm{J} 2$ & 0.029 & 0.029 & 0.013 & 0.013 \\
\hline \multirow{2}{*}{ GMDH(PSS) } & $\mathrm{J} 1$ & 0.08 & 0.037 & 0.017 & 0.017 \\
\cline { 2 - 7 } & $\mathrm{J} 2$ & 0.083 & 0.084 & 0.083 & 0.081 \\
\hline NN & $\mathrm{J} 1$ & 0.119 & 0.133 & 0.108 & 0.094 \\
\cline { 2 - 7 } & $\mathrm{J} 2$ & 0.114 & 0.133 & 0.102 & 0.11 \\
\hline
\end{tabular}

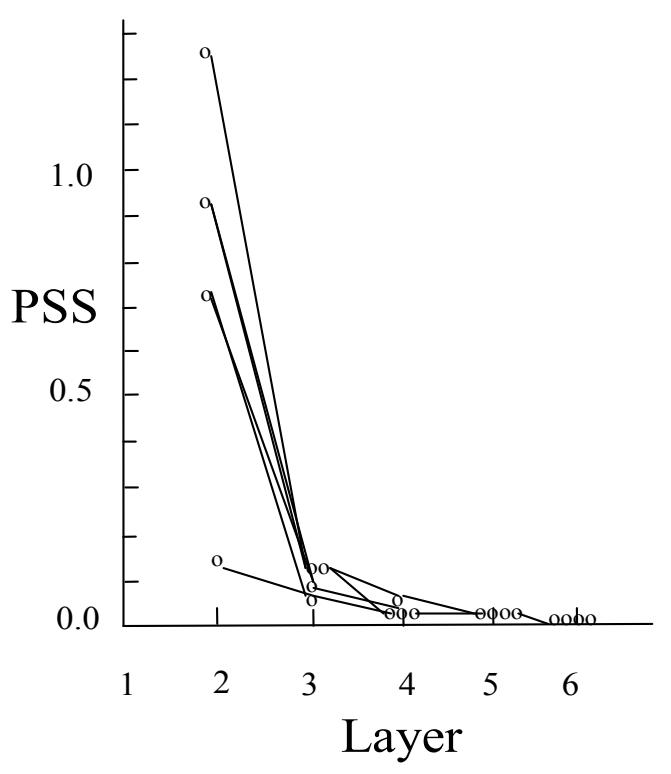

Fig. 5 Variation of PSS values in the layers

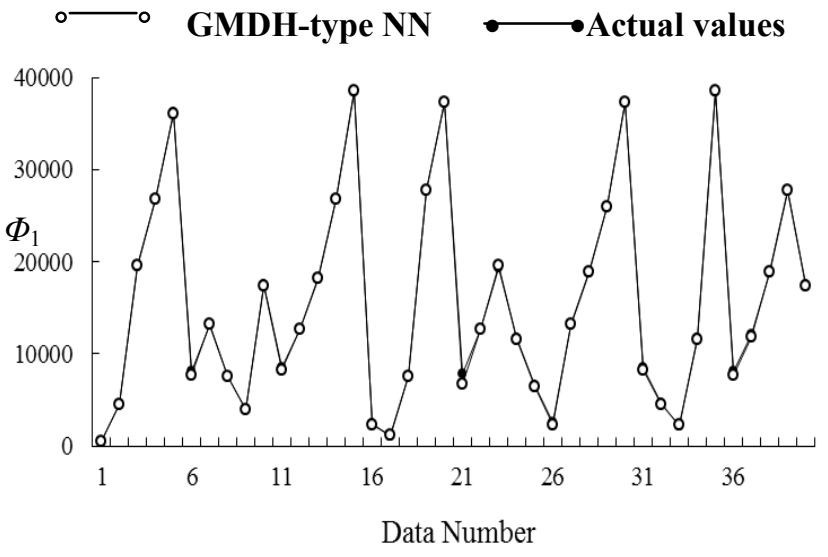

Fig.6 Estimated values of $\phi_{1}$ by revised GMDH-type neural network

\subsection{Comparison of the revised GMDH-type neural network and other models}

The identification results were compared with those of the conventional GMDH-type neural network which is called as feedback GMDH-type neural network, GMDH algorithm, conventional multilayered neural network trained using the back propagation algorithm.

\section{1) Feedback GMDH-type neural network [3]}

We have developed the feedback GMDH-type neural network algorithm and applied to the nonlinear system identification problem [3]. In this algorithm, feedback loop calculations are carried out and the GMDH-type neural network architecture is organized using the feedback loop calculations. In the neuronal calculation, the stepwise regression analysis is used in order to estimate the parameters of the neurons. The values of $J_{1}$ and $J_{2}$ are shown in Table1.

\section{2) GMDH algorithm}

The identification results were referred from [11]. Four input variables were used but the useless input variable $x_{4}$ was automatically eliminated. Four intermediate variables were selected. The calculation was terminated at the fifth layer. The values of $J_{1}$ and $J_{2}$ are shown in Table1.

3) Conventional multilayered neural network trained using the back propagation algorithm

The neural network had three layered structures. Four input variables were used in the input layer and eight neurons were used in the hidden layer. The estimated values of $\phi_{1}$ by the conventional neural network are shown in Fig.7. The values of $J_{1}$ and $J_{2}$ are shown in Table1.

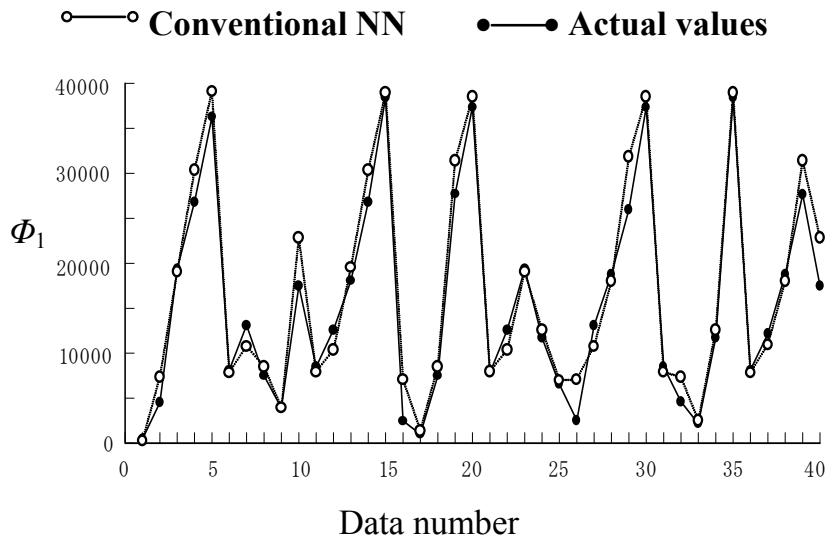

Fig.7 Estimated values of $\phi_{1}$ by the conventional multilayered neural network

\section{Discussions}

From the identification results, we can say the following:

1) Both estimation and prediction errors of the revised GMDH-type neural network were smallest in the four identified models. We can see that the revised GMDH-type neural network is a very accurate identification method for the nonlinear system. 
2) In the revised GMDH-type neural network, PSS values at the second layer were not small but it was gradually decreased through layers and the small PSS values were obtained at the sixth layer.

3) In the revised GMH-type neural network, the complexity of the neural network increases through the layers and the calculation of the revised GMDH-type neural network is terminated when the complexity of the neural network fits the complexity of the nonlinear system. We consider that the nonlinear combinations of the input variables are very important in the complex nonlinear system and the influence of the high order terms of the input variables must be contained in the neural network architectures. In the conventional neural network, the influence of high order terms of the input variables is not considered. Furthermore, it does not have the ability of self-selecting useful input variables. So the accuracy of the neural network was not good.

4) In the revised GMDH-type neural network, many nonlinear combinations of the input variables are generated and the optimum neuron architectures are calculated for these combinations. The learning calculations using the principal component-regression analysis with PSS criterion, are carried out for all neurons. In these learning calculations, the computation times are very short because the sizes of the neuron architectures in Eq,(2), (4), (6), (8), (10) and (12) are very small. The calculation time of the nonlinear system identification in this study is about two seconds. On the other hand, in the conventional sigmoid function neural network, the calculation time of the back propagation algorithm is long and it is about a few minutes in this study, and furthermore, we must repeat the calculations of the back propagation many times in order to fine out the optimum neural network architecture changing the structural parameters of the neural network.

5) The revised GMDH-type neural network can organize the conventional neural network architectures (sigmoid function type and radial basis function type architectures) and the GMDH architecture (polynomial type architecture). This algorithm contains the characteristics of the conventional neural networks and the GMDH algorithm and it is a very flexible method for the identification problem of the complex nonlinear system.

\section{Conclusions}

In this paper, the revised GMDH-type neural network algorithm using the principal component-regression analysis was proposed. This algorithm can automatically organize a multilayered neural network architecture fitting the complexity of the nonlinear system using the heuristic self-organization method. In this algorithm, the parameters of the neurons are estimated using the principal component-regression analysis and the multicolinearity dose not generate. Therefore, very stable multi-layered neural network architecture can be organized. Furthermore, optimum neural network architecture is automatically selected from three types of neural network architectures, such as, sigmoid function neural network, RBF neural network and polynomial neural network. The structural parameters such as the number of hidden layers, the number of neurons in hidden layers and useful input variables are automatically selected to minimize prediction error criterion defined as PSS. This algorithm was applied to the nonlinear system identification and it is shown that revised GMDH-type neural network algorithm is a valuable method for the nonlinear system identification since the neural network architectures are automatically organized using the revised GMDH-type neural network algorithm.

\section{Acknowledgment}

This work was supported by (JSPS) KAKENHI 22560403.

\section{References}

[1] T. Kondo and J. Ueno, "Multi-layered GMDH-type neural network self-selecting optimum neural network architecture and its application to 3-dimensional medical image recognition of blood vessels", International Journal of Innovative Computing, Information and Control, vol.4, no.1, pp.175-187, 2008.

[2] T. Kondo, J. Ueno, "Logistic GMDH-type neural network and its application to identification of X-ray film characteristic curve", Journal of Advanced Computational Intelligence and Intelligent Informatics, vol.11, no.3, pp.312-318, 2007.

[3] T. Kondo and J. Ueno, "Feedback GMDH-type neural network algorithm self-selecting optimum neural network architecture", Proceedings of $12^{\text {th }}$ international symposium on Artificial Life and Robotics, No.GS21-4, pp.1-4, 2007.

[4] T. Kondo, "GMDH neural network algorithm using the heuristic self-organization method and its application to the pattern identification problem", Proc. of the 37th SICE Annual Conference, pp.1143-1148, 1998.

[5] S. J. Farlow ed., Self-organizing methods in modeling, GMDH-type algorithm, New York: Marcel Dekker Inc., 1984.

[6] A. G. Ivakhnenko, "Heuristic self-organization in problems of engineering cybernetics", Automatica, vo.6, no.2, pp.207-219, 1970.

[7] H. Akaike, "A new look at the statistical model identification", IEEE Trans. Automatic Control, vol.AC-19, no.6, pp.716-723, 1974.

[8] H. Tamura, T. Kondo, "Heuristics free group method of data handling algorithm of generating optimum partial polynomials with application to air pollution prediction", Int. J. System Sci., vol.11, no.9, pp.1095-1111, 1980.

[9] N. R. Draper, H. Smith, Applied Regression Analysis, New York: John Wiley and Sons, 1981.

[10] T. Haga and S. Hasimoto, Regression analysis and principal component analysis, Tokyo: Union of Japanese Scientists and Engineers, 1986.

[11] T. Kondo, "GMDH type Neural Network Algorithm Identifying a Network Structure with the Heuristic Self-Organization Method", Trans. ISCIE, Vol.11, No.4, pp.198-207, 1998. 